\title{
Deep H I observations of the compact high-velocity cloud HVC 125+41-207
}

\author{
C. Brüns ${ }^{1,2}$, J. Kerp ${ }^{1}$, and A. Pagels ${ }^{1}$ \\ 1 Radioastronomisches Institut der Universität Bonn, Auf dem Hügel 71, 53121 Bonn, Germany \\ 2 The Australia Telescope National Facility, CSIRO, PO Box 76, Epping NSW 1710, Australia
}

Received 14 December 2000 / Accepted 7 March 2001

\begin{abstract}
We present deep H i observations of the compact high-velocity cloud HVC 125+41-207 using the 100-m Effelsberg telescope. Our goal was in particular to study the warm neutral medium (WNM) in detail. The Effelsberg data reveals a two phase core/halo structure - one component with a velocity width of $F W H M \approx 5 \mathrm{~km} \mathrm{~s}^{-1}$ (Westerbork data show $F W H M \approx 2 \mathrm{~km} \mathrm{~s}^{-1}$, Braun \& Burton 2000) and one with $F W H M \approx 18 \mathrm{~km} \mathrm{~s}^{-1}$. The column density distribution of the warmer component is highly asymmetric and shows a head-tail structure. We performed a Gaussian decomposition of the cloud and found that $52 \%$ of the $\mathrm{H}$ I mass of the cloud is in the WNM. $24 \%$ of the WNM is located in the tail. The overall structure and the systematic variation of the observational parameters radial velocity, velocity dispersion and column density indicate that this cloud is currently interacting with the ambient medium. The Westerbork H I data of this HVC (Braun \& Burton 2000) reveals an interesting dense condensation. Assuming that this condensation is virialized and in pressure equilibrium with the ambient medium, we derive a distance of $130 \mathrm{kpc}$ for HVC 125+41-207. Following these considerations, it is possible to constrain the parameters $n_{\mathrm{IGM}}<7.810^{-6} \mathrm{~cm}^{-3}$ and $T_{\mathrm{IGM}}>1.110^{5} \mathrm{~K}$ of the intergalactic medium of the Local Group.
\end{abstract}

Key words. ISM: clouds, kinematics and dynamics - Galaxy: halo - galaxies: intergalactic medium

\section{Introduction}

High-velocity clouds (HVCs), first discovered by Muller et al. (1963), are defined as neutral atomic hydrogen clouds with radial velocities (relative to the localstandard-of-rest frame, LSR) that can not be explained by simple galactic rotation models. Up to now there is no general consensus on the origin and the basic physical parameters of HVCs. The most critical issue of HVC research is the distance uncertainty. Danly et al. (1993) determined an upper limit of the distance to the Galactic Plane of $z \leq 3.5 \mathrm{kpc}$ for HVC complex M. The most important step forward is the bracket of the distance from the Galactic Plane of $2.5 \leq z \leq 7 \mathrm{kpc}$ for HVC complex A (van Woerden et al. 1999). These results clearly indicate that the HVC complexes $\mathrm{M}$ and $\mathrm{A}$ are located in the gaseous halo of the Milky Way.

Oort (1966) proposed an extragalactic origin for the HVCs. He argued, that the formation of galaxies is still an ongoing process and HVCs represent primordial clouds that are currently accreted by the Milky Way.

Send offprint requests to: C. Brüns, e-mail: cbruens@astro.uni-bonn.de
Blitz et al. (1999) revived the hypothesis that some HVCs are primordial gas left over from the formation of the Local Group galaxies. Braun \& Burton (1999) identified 65 compact and isolated HVCs and argued that this ensemble represents a homogeneous subsample of HVCs at extragalactic distances. Observational evidence for extragalactic HVCs may also be found by the detection of the highly ionized high-velocity gas by Sembach et al. (1999), because of its very low pressure of about $2 \mathrm{~K} \mathrm{~cm}^{-3}$.

Meyerdierks (1991) detected an HVC that appears like a cometary shaped cloud with a central core and an asymmetric envelope of warm neutral atomic hydrogen. He interpreted this head-tail structure as the result of an interaction between the HVC and normal galactic gas at lower velocities. Towards HVC-complex C, Pietz et al. (1996) discovered the so-called H I "velocity bridges" which seem to connect some HVCs with the normal rotating interstellar medium. The most straight forward interpretation for the existence of such structures is to assume that a fraction of the HVC gas was stripped from the main condensation. Brüns et al. (2000a) extended the investigations of Meyerdierks (1991) and Pietz et al. (1996) over the entire sky covered by the new Leiden/Dwingeloo H I 
21-cm line survey (Hartmann \& Burton 1997) and found head-tail structures in all HVC-complexes except for the very faint HVC-complex L. Their analysis revealed that the absolute value of the radial velocity of the tail is always lower than the value for the head of the HVC $\left(\left|v_{\mathrm{LSR}, \text { tail }}\right|<\left|v_{\mathrm{LSR}, \text { head }}\right|\right)$. In addition, it was shown that the fraction of HVCs showing a head-tail structure increases proportional to the peak column density and increasing radial velocity $\left|v_{\mathrm{GSR}}\right|$.

In this letter, we present deep integrated Effelsberg H I 21-cm line data of the compact high-velocity cloud HVC 125+41-207 (discovered by Hulsbosch \& Wakker 1988), which was placed at extragalactic distances by Braun \& Burton (2000). In Sect. 2, we give a brief summary of the observational parameters. The observations show that HVC $125+41-207$ is a head-tail HVC. The results - including a Gaussian decomposition of the spectra - are presented in Sect. 3. We summarize and discuss the results of the Gaussian decomposition and interpret the head-tail structure as an interaction with an ambient medium in Sect. 4. In addition we derive the distance to HVC 125+41-207 and constrain the parameters $n$ and $T$ of the intergalactic medium.

\section{Observations}

The observations were carried out between June and October 2000 at the 100-m Effelsberg telescope using the $21-\mathrm{cm}$ receiver $\left(T_{\text {sys }} \approx 24 \mathrm{~K}\right)$. The HPBW at $21-\mathrm{cm}$ is $9^{\prime}$. The 1024 channel autocorrelator was splitted into two banks of 512 channels for each polarization. The bandwidth of $1.5 \mathrm{MHz}$ offers a velocity resolution of $0.65 \mathrm{~km} \mathrm{~s}^{-1}$. The HVC was mapped on a regular grid in equatorial coordinates with grid-spacings of 4.5 arcmin and an integration time of 4 min per position. The standard calibration source S7 was used for the flux-calibration. We subtracted a third order polynomial for the baseline correction. After averaging the two polarizations we reach an rms-noise of $0.09 \mathrm{~K}$ at full velocity resolution of $0.65 \mathrm{~km} \mathrm{~s}^{-1}$.

Radio frequency interference was reduced significantly during the last two years at the Effelsberg telescope. There is no detectable interference within the used bandwidth.

\section{Results}

Figure 1a shows the column density distribution of the compact high-velocity cloud HVC $125+41-207$. Black contour lines represent the Effelsberg observations. The lowest contour line indicates a column density of $310^{18} \mathrm{~cm}^{-2}$. This corresponds to $3 \sigma$ significance level. The peak column density in this map is $1.210^{20} \mathrm{~cm}^{-2}$. The distribution of the low column density material is highly asymmetric. While there is a sharp column density gradient on the eastern side, the column density on the western side drops off very slowly. This kind of morphology is called head-tail structure (Meyerdierks 1991; Brüns et al. 2000a).

\subsection{Gaussian decomposition}

Figure 1c shows a typical H i spectrum containing one component with a low and one with a high velocity dispersion. The two component structure is visible in all spectra with column densities $N_{\mathrm{H}_{\mathrm{I}}} \geq 210^{19} \mathrm{~cm}^{-2}$, consistent with HVC-models (Wolfire et al. 1995) and previous observations.

We performed a Gaussian decomposition of the Effelsberg dataset to study the two gas-phases in detail. Each spectrum was inspected individually if a single Gaussian fits the shape of the line sufficiently. If not, two Gaussians were fitted to the spectrum. In the very end of the tail $\left(\alpha_{2000}<12^{\mathrm{h}} 05^{\mathrm{m}}\right)$ neighbouring spectra were averaged and hanning smoothed to gain a higher signalto-noise ratio.

\subsection{The column density distribution}

The Gaussian decomposition gives the opportunity to separate the large and the low velocity dispersion components. The low velocity dispersion component is concentrated in two main condensations, clump I centred on $\left(\alpha_{2000}=12^{\mathrm{h}} 27^{\mathrm{m}} 20^{\mathrm{s}}, \delta_{2000}=75^{\circ} 25^{\prime} 30^{\prime \prime}\right)$ and clump II centred on $\left(\alpha_{2000}=12^{\mathrm{h}} 22^{\mathrm{m}} 30^{\mathrm{s}}, \delta_{2000}=75^{\circ} 31^{\prime} 30^{\prime \prime}\right)$. The two clumps have similar peak column densities $\left(N_{\mathrm{H} \text { I }} \approx\right.$ $\left.810^{19} \mathrm{~cm}^{-2}\right)$.

The column density distribution of the component with the large velocity dispersion also shows two maxima, but shifted in respect to the cold gas-phase (see Fig. 2a). The column density distribution is highly asymmetric, in the tail it decreases exponentially $\left(\mathrm{e}^{-\alpha / \tau}\right.$ with $\tau=0.515 \pm 0.014$ ). The last four data points deviate significantly from an exponential decay.

The slice perpendicular to the cloud (Fig. 2b) shows that the warm component is smoother distributed and more extended $\left(F W H M=18^{\prime}\right)$ than the cold component $\left(F W H M=11^{\prime}\right)$. The Westerbork data (Fig. 1b) show, that the extent of the cold component $\left(F W H M=6^{\prime}\right)$ is much smaller at high angular resolution.

The total Hi mass can by calculated by summing up all Gaussian components over the extent of the cloud and assuming a distance. The $\mathrm{HI}$ mass scales quadratically with distance. The total $\mathrm{HI}_{\mathrm{I}}$ mass is $M_{\mathrm{HI}}=$ $2.410^{6}(\mathrm{~d} / 200 \mathrm{kpc})^{2} M_{\odot}$. The component with the large velocity dispersion accounts for $52 \%$ of the total H I mass. $24 \%$ of this material ( $12.5 \%$ of the total H I mass) is located in the tail $\alpha_{2000}<12^{\mathrm{h}} 12^{\mathrm{m}}$.

\subsection{The distribution of temperature and turbulence}

Obviously, there are two gas-phases, one centered at $F W H M=5 \mathrm{~km} \mathrm{~s}^{-1}$ and one centered at $F W H M=$ $18 \mathrm{~km} \mathrm{~s}^{-1}$. This corresponds to Doppler temperatures of $T_{\mathrm{D}}=540 \mathrm{~K}$ and $T_{\mathrm{D}}=7000 \mathrm{~K}$. These are only upper limits to the kinetic temperatures, as unresolved small scale structure and turbulence also contribute to the Doppler temperature. The Westerbork observations from 

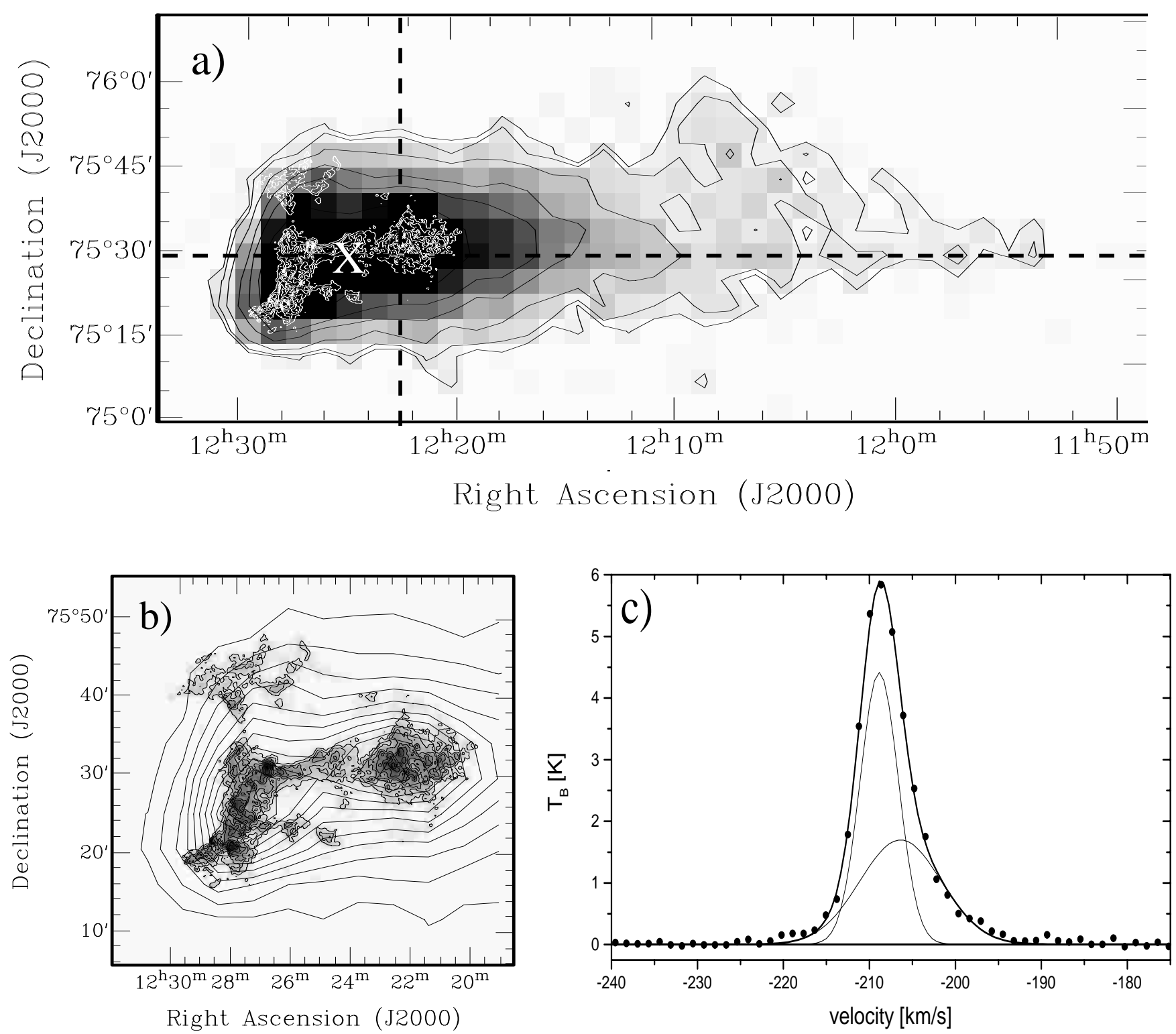

Fig. 1. a) Column density distribution of HVC $125+41-207$. The black contour levels represent $3,5,10,20,30,50,10010^{18} \mathrm{~cm}^{-2}$ as observed with the Effelsberg telescope. The white contour levels represent the Westerbork data from Braun \& Burton (2000) starting from $5010^{18} \mathrm{~cm}^{-2}$ in steps of $5010^{18} \mathrm{~cm}^{-2}$. The distribution of the Effelsberg H i data show a head-tail structure. The dashed lines represent the slices through the cloud (see Fig. 2). The "X" indicates the position of the spectrum shown in Fig. 1c. b) Column density distribution of the Westerbork data with same contour levels as in Fig. 1a. The Effelsberg data are represented by contour levels starting with $510^{18} \mathrm{~cm}^{-2}$ increasing in steps of $1010^{18} \mathrm{~cm}^{-2}$. c) This figure shows a typical $\mathrm{H}$ I spectrum containing a warm and a cold component (represented by the two Gaussians). The points represent the data, the thick line is the fit to the spectrum. Note that the warm gas-phase is at a lower radial velocity than the cold component

Braun \& Burton (2000) show, that the compact cores have velocity dispersions of the order $F W H M=2 \mathrm{~km} \mathrm{~s}^{-1}$ or $T_{\mathrm{D}}=85 \mathrm{~K}$ at an angular resolution of $28^{\prime \prime}$. A detailed analysis of the small-scale structure is not possible with the Effelsberg data. Nevertheless, the comparison of the Westerbork and Effelsberg data (Fig. 1b) shows that the general distribution of the cold component can be studied, especially with respect to velocity gradients (see Sect. 3.4).

The warm component is not affected by unresolved small-scale structure. However, the data can not distinguish between an increase in turbulence or temperature.
The velocity dispersion of the warm gas-phase is increasing from $F W H M \approx 10 \mathrm{~km} \mathrm{~s}^{-1}$ to $F W H M \approx 20 \mathrm{~km} \mathrm{~s}^{-1}$ over the extend of the cloud and remains approximately constant in the tail (see Fig. 2c). This corresponds to an increasing Doppler temperature from $T_{\mathrm{D}}=2000 \mathrm{~K}$ to $T_{\mathrm{D}}=10000 \mathrm{~K}$ in the tail. The slice perpendicular to the cloud shows, that the velocity dispersion increases significantly towards the border of the cloud (see Fig. 2d). For this particular slice, the Doppler temperature increases from $T_{\mathrm{D}} \approx 5000 \mathrm{~K}$ in the centre to $T_{\mathrm{D}} \approx 11000 \mathrm{~K}$ at the border of the cloud. 

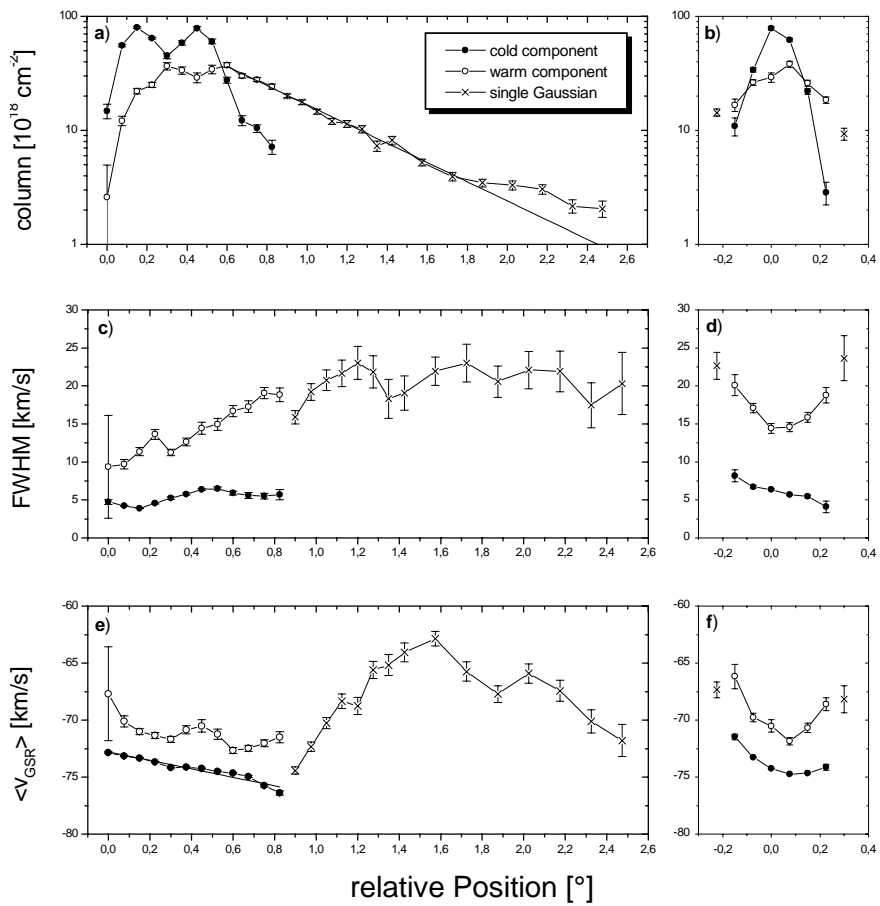

Fig. 2. Results of the Gaussian decomposition of the H I spectra. The cold gas-phase is represented by filled circles, the warm gas-phase by open circles. Results from a single Gaussian fit are represented by crosses. The diagrams a),c) and e) show the results for a slice along the tail, the diagrams $\mathbf{b}$ ),d) and f) represent the slice perpendicular to the orientation of the tail. The line in diagram a) is an exponential fit to the data. The line in diagram e) is a fit to the velocity field

\subsection{The velocity field}

The interpretation of the velocity gradients in the localstandard-of-rest frame is difficult, because the angle between the solar velocity vector and the line of sight varies over the extend of the cloud and therefore introduces a velocity gradient that is not related to the $\mathrm{HVC}$ itself. The effect is of the order $2 \mathrm{~km} \mathrm{~s}^{-1}$ per degree and can be removed by converting the velocity into the galacticstandard-of-rest frame.

Figure 2e shows the parameter mean velocity $\left(v_{\mathrm{GSR}}\right)$ along a slice in direction of the tail. There is a regular velocity gradient in the cold phase. This gradient can be easily explained by the same type of projection effect that urged us to convert the radial velocity into the galacticstandard-of-rest-frame: the angle between the line of sight and the HVC velocity vector is changing over the extend of the HVC. This effect can be used to derive the threedimensional velocity of the HVC. The gradient should be of the form $v_{\mathrm{GSR}}=v_{\text {total }} \cos \left(\alpha+\alpha_{0}\right)$. The result of a least-squares fit is also shown in Fig. 2e. The total spacevelocity is $v_{\text {total }}=225 \pm 18 \mathrm{~km} \mathrm{~s}^{-1}$ and the angle between the line of sight and the HVC velocity vector is $\alpha_{0}=$ $108.4 \pm 1.5$, i.e. the direction of proper motion is almost perpendicular to the line of sight in the direction of the sharp velocity gradient. This result indicates, that the tail is trailing the HVC.
The spectrum in Fig. 1c shows, that the warm component has a lower radial velocity than the cold component. It is remarkable, that this is true for all spectra where a two component fit was feasible. This result indicates that the two gas-phases are separating from each other. The difference between the radial velocities of the warm and the cold component is minimal where the column density of the warm gas-phase has its maxima.

The absolute value $\left|v_{\text {LSR }}\right|$ of the radial velocity is steeply decreasing in the first part of the tail and increasing towards the end (Fig. 2e). The slice perpendicular to the cloud shows, that $\left|v_{\text {LSR }}\right|$ decreases significantly towards the borders of the cloud (see Fig. 2f).

\section{Summary and discussion}

Our Effelsberg H I observations of HVC 125+41-207 show, that the compact HVC has a cometary appearance. The $\mathrm{HI}$ gas gets warmer and/or more turbulent to the $\mathrm{HI}$ boundaries of the cloud as well as towards the tail. The radial velocity $\left|v_{\text {LSR }}\right|$ of the $\mathrm{H}$ I gas is decreasing towards the border of the cloud and in the tail. It is remarkable that the warm gas-phase always shows lower $\left|v_{\mathrm{LSR}}\right|$ than the cold phase.

These results demonstrate that HVC $125+41-207$ is definitively not a relaxed cloud in equilibrium. The observational results suggest that the warm gas-phase is stripped off the main body of the HVC. This kind of behaviour is for instance expected from a ram-pressure interaction: the diffuse warm gas has a much larger crosssection for the ram-pressure interaction than the small, dense clumps of the cold medium.

Santillan et al. (1999) for instance performed MHD simulations to explain head-tail HVCs at $z$-height of a few kpc. They were successful in explaining head-tail structures. The tail consists of material that was stripped off the main body (i.e. the head) of the cloud. Their models showed that the evolution of the shocked layer generates a tail that oscillates, creating vorticity and turbulent flows along its trajectory. This explains the velocity structure in the tail of HVC $125+41-207$ with increasing $\left|v_{\text {LSR }}\right|$ to the end of the tail.

It is quite easy to produce a head-tail structure in the lower Galactic halo. Nevertheless, it might be possible to explain the existence of head-tail structures even in the intergalactic space of the local group. Brüns et al. (2000a, 2000b) showed that there are head-tail HVCs in the Magellanic Stream and the Leading Arm. New magnetohydrodynamical simulations considering the critical velocity effect (Konz, priv. comm.) explain head-tail structures if the ambient medium has a plasma density of the order $n \approx 10^{-5} \mathrm{~cm}^{-3}$ and a magnetic field $|B|$ of the order of a few $\mu \mathrm{G}$.

The Westerbork data (Fig. 1b) show one remarkable condensation $\left(\alpha_{2000}=12^{\mathrm{h}} 26^{\mathrm{m}} 50^{\mathrm{s}}, \delta_{2000}=75^{\circ} 31^{\prime} 26^{\prime \prime}\right)$ with a brightness temperature $T_{\mathrm{B}}=75 \mathrm{~K}$ and a velocity width of $F W H M=2 \mathrm{~km} \mathrm{~s}^{-1}$, i.e. a Doppler temperature of $T_{\mathrm{D}}=85 \mathrm{~K}$. This clump has therefore a 
kinetic temperature $T_{\text {kin }} \approx 80 \mathrm{~K}$. The clump has an apparent column density of $N_{\mathrm{H} \text { I }}=4.510^{20} \mathrm{~cm}^{-2}$, but due to optical depth effects a real column density of the order $N_{\mathrm{H} \text { I }} \approx 110^{21} \mathrm{~cm}^{-2}$. We derive a distance to HVC $125+41-$ 207 assuming that the clump is virialized and in pressure equilibrium with the ambient medium. If we consider an isothermal sphere of uniform density without magnetic fields, we can use the equation (Spitzer 1978)

$4 \pi R^{3} P_{0}=\frac{3 M k T}{\mu}-\frac{3 G M^{2}}{5 R}$

$R$ is the radius, $M$ the mass and $T$ the temperature of the sphere. $P_{0}$ is the pressure of the ambient medium and $\mu$ the mean mass per particle within the sphere. The mass $M$ is given by $M=4 / 3 \pi R^{3} \rho$, with $\rho=\mu N_{\mathrm{H} \mathrm{I}}(2 R)^{-1}$, where the column density of the clump is $N_{\mathrm{HI}}=110^{21} \mathrm{~cm}^{-2}$. The radius $R=0.5 \theta d$ is calculated by the observed angular diameter of the clump $\theta=90^{\prime \prime}$ and the distance $d$. The external pressure is $P_{0}=k T_{\mathrm{w}} N_{\mathrm{H} \text { I, }} d^{-1} \theta_{\mathrm{w}}^{-1}$, where the column density is $N_{\mathrm{H} \text {, w }}=210^{19} \mathrm{~cm}^{-2}$, the temperature is $T_{\mathrm{w}}<3000 \mathrm{~K}$, and the angular diameter of the ambient medium is $\theta_{\mathrm{w}}=18^{\prime}$. Inserting into Eq. (1) and solving for the distance $d$ gives

$d=\frac{15 k\left(N_{\mathrm{H}}{ }_{\mathrm{I}} T-\theta \theta_{\mathrm{w}}^{-1} N_{\mathrm{H}, \mathrm{w}} T_{\mathrm{w}}\right)}{G \pi \theta N_{\mathrm{H}}^{2} \mu^{2}}$.

The unknown values in Eq. (2) are $T_{\mathrm{w}}$ and $\mu$. Assuming $\mu=1.25 m_{\mathrm{H}}$, i.e. including the normal amount of Helium, but without any molecular matter, we get possible distances between $d\left(T_{\mathrm{w}}=3000 \mathrm{~K}\right)=126 \mathrm{kpc}$ and $d\left(T_{\mathrm{w}}=\right.$ $100 \mathrm{~K})=134 \mathrm{kpc}$. Any molecular gas content would lower the derived distance, e.g. $\mu=2 m_{\mathrm{H}}$ gives $d \approx 50 \mathrm{kpc}$.

The density of the ambient WNM at $d=130 \mathrm{kpc}$ is $n_{\mathrm{w}}=9.810^{-3} \mathrm{~cm}^{-3}$ with pressure $P_{0}<29.5 \mathrm{~K} \mathrm{~cm}^{-3}$.

In the remaining part of this section, we will discuss implications on the intergalactic medium (IGM). Our Effelsberg data demonstrate, that there must be an ambient medium, which is responsible for the head-tail structure. The observed pressure is $P_{0}=P_{\text {ram }}+P_{\mathrm{IGM}}$. The ram pressure is $P_{\text {ram }}=0.5 \rho_{\mathrm{IGM}} v^{2}=P_{0}-P_{\mathrm{IGM}}<$ $30 \mathrm{~K} \mathrm{~cm}^{-3}$. An upper limit to the density of the ambient medium $\rho_{\text {IGM }}$ can be calculated using the space velocity of $v=225 \mathrm{~km} \mathrm{~s}^{-1}$ determined in Sect. 3.4: $\rho_{\text {IGM }}<1.610^{-32} \mathrm{~kg} \mathrm{~cm}{ }^{-3}=240 M_{\odot} \mathrm{kpc}^{-3}$. With $\rho_{\mathrm{IGM}}=1.25 m_{\mathrm{H}} n_{\mathrm{IGM}}$ follows that $n_{\mathrm{IGM}}<7.810^{-6} \mathrm{~cm}^{-3}$.

Assuming that the pressure of $2 \mathrm{~K} \mathrm{~cm}^{-3}$, derived by Sembach et al. (1999) for highly ionized high-velocity clouds, is valid for the IGM in general, the temperature of the IGM can be calculated. Using $P k^{-1}=2.3 n_{\mathrm{IGM}} T_{\mathrm{IGM}}$, where 2.3 is the number of particles per hydrogen atom for an ionized medium including helium, gives $T_{\mathrm{IGM}}>$ $1.110^{5} \mathrm{~K} . T_{\text {IGM }}$ is sufficiently high to keep the IGM ionized (neutral gas would have been detected in absorption against quasars) and low enough to be undetectable in X-rays.
However, the derived distance of $130 \mathrm{kpc}$ is based on the assumption of equilibrium and no molecular gas content. As the HVC is obviously interacting with the ambient medium, equilibrium conditions are not really justified. The HVC could be located at larger distances having a collapsing clump that might form stars in future - at a distance of a Mpc the Hi mass is about 40 times larger than the virial mass. On the other hand, if the clump contains a significant amount of molecular matter, this will localize the HVC in the Galactic Halo.

Our observations demonstrated that both, deep integrated observations of the warm neutral medium and high resolution observations of the cold neutral medium are necessary to obtain further insights to the nature of HVCs. Deep H I observations of a number of compact HVCs are planed for 2001. These observations will help to determine general parameters in compact HVCs.

Acknowledgements. Part of this work was supported by the German Deutsche Forschungsgemeinschaft, DFG project number ME 745/19. We thank our referee W. B. Burton for constructive comments and R. Braun \& W. B. Burton for kindly providing their Westerbork data.

\section{References}

Blitz, L., Spergel, D. N., Teuben, P. J., Hartmann, D., \& Burton, W. B. 1999, ApJ, 514, 818

Braun, R., \& Burton, W. B. 1999, A\&A, 341, 437

Braun, R., \& Burton, W. B. 2000, A\&A, 354, 853

Brüns, C., Kerp, J., Kalberla, P. M. W., \& Mebold, U. 2000a, A\&A, 357, 120

Brüns, C., Kerp, J., \& Staveley-Smith, L. 2000b, in Mapping the Hidden Universe: The Universe Behind the Milky Way - The Universe in H I, ed. R. C. Kraan Korteweg, \& P. A. Henning, ASP Conf., 218, 349

Danly, L., Albert, C. E., \& Kuntz, K. D. 1993, ApJ, 416, L29

Hartmann, D., \& Burton, W. B. 1997, An atlas of Galactic Neutral Hydrogen Emission (Cambridge University Press) Hulsbosch, A. N. M., \& Wakker, B. P. 1988, A\&AS, 75, 191 Meyerdierks, H. 1991, A\&A, 251, 269

Muller, C. A., Oort, J. H., \& Raimond, E. 1963, C.R. Acad. Sci. Paris, 257, 1661

Oort, J. H. 1966, Bull. Astr. Inst. Netherlands, 18, 421

Pietz, J., Kerp, J., Kalberla, P. M. W., et al. 1996, A\&A, 308, L37

Santillan, A., Franco, J., Martos, M., \& Kim, J. 1999, ApJ, 515,657

Sembach, K. R., Savage, B. D., Lu, L., \& Murphey, E. M. 1999, ApJ, 515, 108

Spitzer, L. 1978, Physical Processes in the interstellar medium (Wiley, New York)

van Woerden, H., Schwarz, U. J., Peltier, R. F., Wakker, B. P., \& Kalberla, P. M. W. 1999, Nature, 400, 138

Wolfire, M. G., McKee, C. F., Hollenbach, D., \& Tielens, A. G. G. M. 1995, ApJ, 453, 673 